\title{
CT texture analysis of abdominal lesions - Part II: Tumors of the Kidney and Pancreas
}

\section{IMAGING}

\section{REVIEW ARTICLE}



IMAGING 13 (2021) 1, 25-36

DOI: $10.1556 / 1647.2021 .00020$

(C) 2021 The Author(s)

${ }^{\dagger}$ Both authors contributed equally to this paper

*Corresponding author. Korányi Sándor str. 2, H-1083 Budapest, Hungary. Tel.: +36 (1) 459-1500 x.61628.

E-mail: kaposi.pal@med. semmelweis-univ.hu

\author{
VERONICA FRANK $^{\dagger} \odot$, SONAZ SHARIATI ${ }^{\dagger}$, \\ BETTINA KATALIN BUDAI $\odot$, BENCE FEJÉR, \\ AMBRUS TÓTH $\odot$, VINCE ORBÁN, VIKTOR BÉRCZI ๑ and \\ PÁL NOVÁK KAPOSI*
}

Department of Radiology, Medical Imaging Centre, Semmelweis University, Faculty of Medicine, Budapest, Hungary

Received: September 24, 2020 • Accepted: April 20, 2021

\begin{abstract}
It has been proven in a few early studies that radiomic analysis offers a promising opportunity to detect or differentiate between organ lesions based on their unique texture parameters. Recently, the utilization of CT texture analysis (CTTA) has been receiving significant attention, especially for response evaluation and prognostication of different oncological diagnoses. In this review article, we discuss the unique ability of radiomics and its subfield CTTA to diagnose lesions in the pancreas and kidney. We review studies in which CTTA was used for the classification of histology grades in pancreas and kidney tumors. We also review the role of radiogenomics in the prediction of the molecular and genetic subtypes of pancreatic tumors. Furthermore, we provide a short report on recent advancements of radiomic analysis in predicting prognosis and survival of patients with pancreatic and renal cancers.
\end{abstract}

\section{KEYWORDS}

radiomics, texture analysis, machine learning, abdominal imaging, kidney, pancreas

\section{Introduction}

Computed tomography (CT) is an efficient non-invasive diagnostic tool capable of lesion detection and differentiation, and it is extensively used in all imaging departments to evaluate oncology patients. Nowadays, precise diagnostics, management and follow-up of oncology patients commonly require repeated imaging sessions. The clinical management of these patients would greatly benefit from automated image analysis methods, which can extract objective and reproducible image parameters from selected regions of interest (ROI) and analyze them with computational tools [1].

The basic concept of radiomic analysis is to generate a large number of quantitative imaging biomarkers from radiology images that can be linked to various phenotypes of the imaged lesions. The identification of the suspected lesion and its boundaries used for labeling the ROI is the first important step (Fig. 1). The radiomic features from the ROIs are used for comparing lesions to detect differences in shape, texture and gray-level distributions in the original radiologic images (Fig. 2). These biomarkers carry important prognostic and diagnostic information and can be used to build prediction models for lesion classification [2].

Radiomic methods may eventually be able to characterize tumor biology by finding links between imaging biomarkers and underlying pathology in radiologic images. This presents a cost-effective approach that could potentially allow us to differentiate benign lesions from malignant ones or determine the aggressiveness and responsiveness of a particular lesion. In this way, radiomics can be employed as a diagnostic and prognostic tool in personalized medicine [3]. Consequently, radiomics may ultimately allow for better clinical decisionmaking leading to more efficient utilization of healthcare resources [4]. 


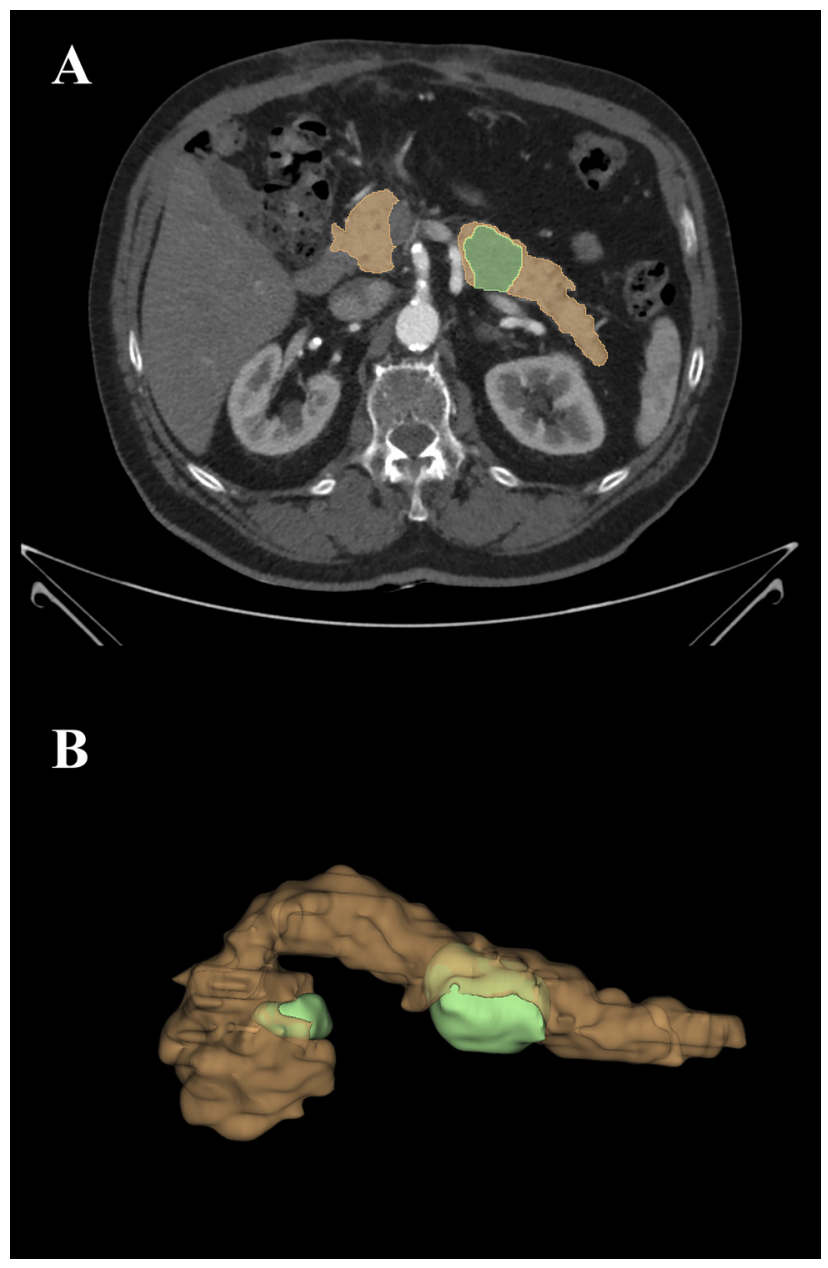

Fig. 1. Manual three-dimensional segmentation of the pancreas parenchyma and pancreatic adenocarcinomas on arterial phase axial CT scan.

Slice-wise manual segmentation of the area of the whole pancreas on the axial view of arterial phase CT scans (A).

Three-dimensional reconstruction of the delineated pancreatic tumors and tumor-free parenchyma (B)

In this review article, we focus on a particular subfield of radiomics, which assesses the texture of the selected area on CT scans mathematically. CT texture analysis (CTTA) extracts quantitative image features, which describe spatial variants of pixel intensities that otherwise cannot be detected [5]. The texture variations may be subtle and contain information on lesions' heterogeneity that cannot be recognized solely by visual inspection. Thus, CTTA may expand the diagnostic potential of conventional imaging studies. Radiomics is often used in combination with machine learning and artificial intelligence (AI) as well, which has attracted increasing attention in the past decade in the field of radiology research (Fig. 3).

\section{Radiomic analysis of pancreatic tumors}

Tumor heterogeneity is an inherent characteristic of malignant lesions containing multiple clones of tumor cells with divergent phenotypic traits, including growth rate, angiogenesis factors, and interactions with the microenvironment $[6,7]$. The histological evaluation of tumors with biopsy allows direct visualization of the tissue architecture, but it is associated with procedural risks and does not describe regional differences inside the lesion. Whereas, imaging studies are non-invasive, have minimal risk of complications, can visualize the entire lesion and enable the assessment of temporal variations. Cross-sectional imaging, especially CT, has excellent spatial resolution and allows for assessment of heterogeneity inside a single-slice or the whole volume of a selected lesion. CT is often the primary imaging modality used for oncological investigations thanks to its high resolution, speed and generally good availability. Nevertheless, the radiological assessment of tumor heterogeneity has mainly been qualitative until recently when automated tools of quantitative assessments have been introduced to clinical practice.

\section{Differentiation between various types of pancreatic lesions with CTTA}

Currently, CT is the standard tool used in the diagnostics of pancreatic diseases as it can differentiate the tumor from the surrounding parenchyma and can precisely assess its extent. However, conventional CT evaluation methods have limited capability to detect and differentiate various phenotypes of pancreatic tumors. CTTA is a novel and rapidly emerging technique that has shown promising results in the detailed characterization of pancreatic lesions (Table 1). In a retrospective case-control study, Chu et al. [8] were able to differentiate between cases with pancreatic ductal adenocarcinoma (PDAC) and healthy controls with normal pancreas. The authors identified 190 patients with PDAC (tumor size $=4.1 \pm 1.7 \mathrm{~cm}$ ) and 190 healthy kidney donors without known pancreatic disease. The area of the whole pancreas in all three dimensions was manually segmented, and 478 radiomic features were extracted. After feature selection, 40 parameters were used for training a random forest (RF) classifier. The binary classifier achieved an excellent overall accuracy with $99.2 \%$, while the area under the curve (AUC) in a receiver operating characteristics (ROC) analysis was 0.999 , with a sensitivity of $100 \%$ and a specificity of $98.5 \%$.

In a recent study by Ren et al. [9]; three-dimensional CTTA of arterial and portal venous phase CT scans showed great potential in differentiating between mass forming pancreatitis and PDAC. In a multivariable logistic regression analysis, five radiomic parameters - surface area, percentile 40, inverse difference moment (IDM), long run emphasis (gray-level run-length matrix feature - GLRLM) and uniformity - extracted in the arterial phase and four texture features - long run emphasis, voxel value sum, long run emphasis and gray level run-length matrix (GLCM) entropy - extracted on the portal venous phase CT scans were proved to be independent predictors of malignant disease. A multivariable logistic regression model constructed from the abovementioned arterial phase features 

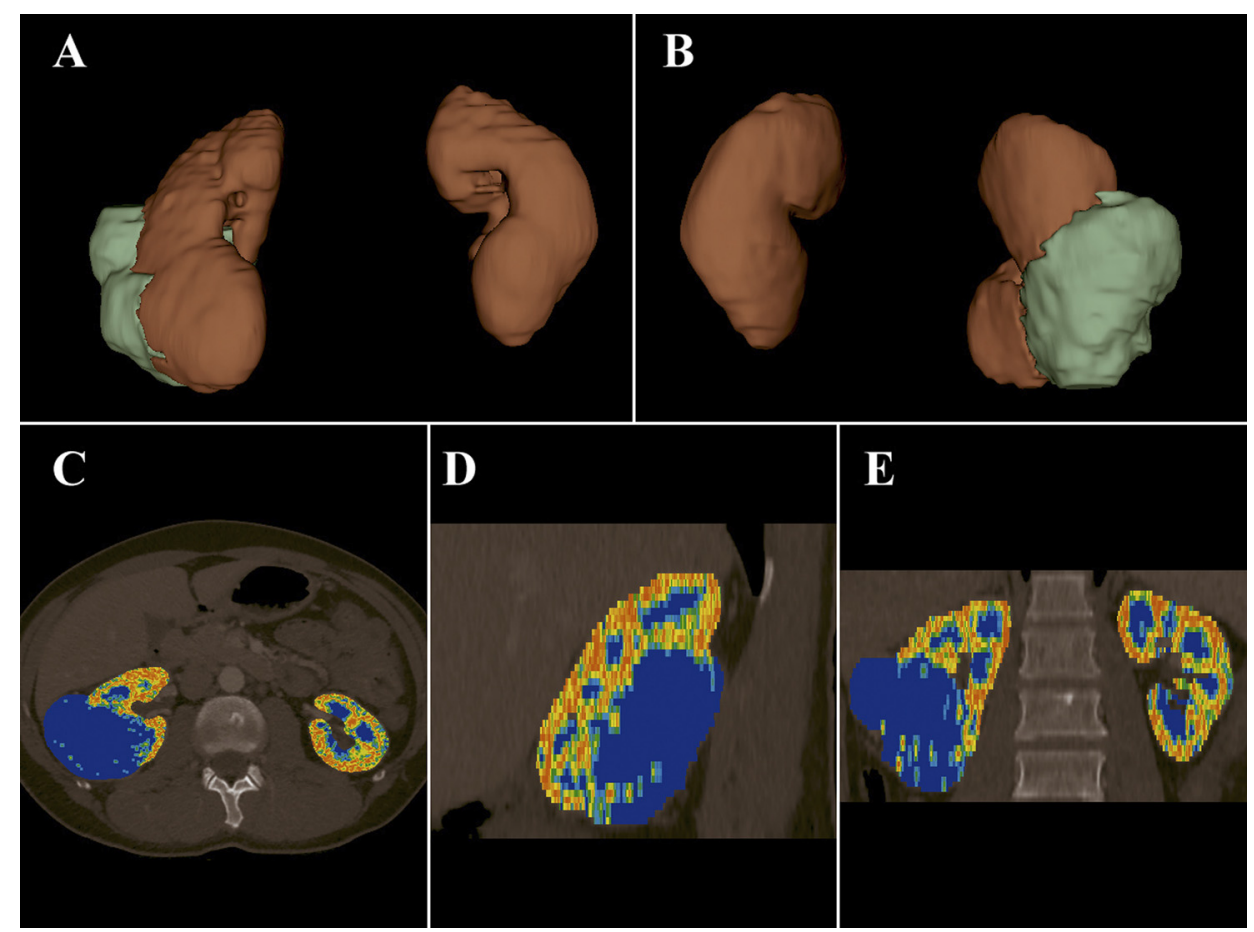

Fig. 2. Textural features of a renal cell carcinoma compared with healthy parenchyma on the arterial phase CT scan.

Anterior view (A) and posterior view (B) of the three-dimensional reconstruction of the manually segmented kidneys and renal tumor. Feature map of gray-level co-occurrence matrix-based texture parameter (Energy) in axial (C), sagittal (D) and coronal (E) view.

*Central Illustration

(Model 2) showed better accuracy than a similar prediction model constructed from portal venous features (Model 3) or a prediction model built from standard imaging features such as arterial phase attenuation and penetrating duct sign (Model 1). The diagnostic performance of the three prediction models determined was AUC $=0.84[95 \%$ confidence interval $(\mathrm{CI})=0.75-0.92]$ (accuracy of $72 \%$ ) for Model 1, AUC $=0.96$ [95\% CI $=0.93-1.0]$ (accuracy of 87\%) for Model 2, and AUC $=0.93$ [95\% CI $=0.87-0.98]$ (accuracy of $89 \%$ ) for Model 3, while an integrated prediction model combining these three models was even more accurate with AUC $=0.98[95 \% \mathrm{CI}=0.92-1.0]$ and accuracy $=94 \%$.

Previous studies used radiomic analysis to differentiate between PDACs and pancreatic neuroendocrine tumors (pNET) and showed promising results. Reinert et al. [10] demonstrated that CTTA of portal venous phase scans could be used for differentiating between pancreas PDAC and pNET with better accuracy than with visual assessment or calculating the tumor-to-parenchyma attenuation ratio. At the same time, Li et al. [11] used three-dimensional CTTA to differentiate atypical pNET from PDACs. In their study, first-order radiomic parameters were extracted on the preoperative portal venous phase CT scans. Mean, median and lower percentiles such as $5^{\text {th }}, 10^{\text {th }}$ and $25^{\text {th }}$ percentiles were significantly lower, while skewness was statistically higher in PDAC than in pNET. In a ROC-AUC analysis, the $5^{\text {th }}$ percentile had higher AUC value $(\mathrm{AUC}=0.811 ; 95 \%$ $\mathrm{CI}=0.704-0.892)$ than mean, skewness, kurtosis and entropy; but the combination of $5^{\text {th }}$ percentile and skewness showed the highest diagnostic performance with an AUC of 0.887 [95\% CI $=0.793-0.948]$.

\section{Prediction of histology grade with CTTA}

Several studies have successfully tested CTTA for the prediction of histology grade in various types of cancers $[12,13]$. The category of pNET is a heterogeneous group, which includes well-differentiated neuroendocrine tumors and poorly differentiated malignant neuroendocrine carcinomas. The World Health Organization classified these tumors into groups based on the Ki-67 index and mitotic activity. Well-differentiated neuroendocrine neoplasms can be divided into three categories: grades 1, 2 and 3. Poorly differentiated neuroendocrine tumors, called neuroendocrine carcinoma grade 3, can be further categorized into small cell and large cell types. As these subgroups show important differences in their pathologic, functional and clinical features, the correct classification can influence the management and improve the prognosis of patients [14].

Previous studies showed that CTTA might offer promise for the non-invasive assessment of histology grade of pancreatic lesions (Table 2). Choi et al. [15] were the first who studied the potential of CTTA for the prediction of histology grade of pNETs. In their study, arterial and portal venous phase CT scans of 66 patients were collected. A twodimensional and a three-dimensional texture analysis were performed using either the largest cross-sectional area of the tumor or the whole tumor volume. In both the $2 \mathrm{D}$ and $3 \mathrm{D}$ analysis of the arterial phase scans, high grade (grade 2 and 
A

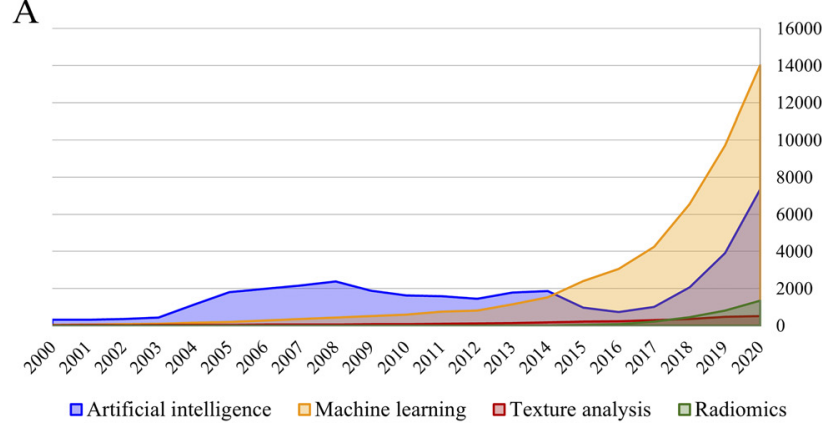

B

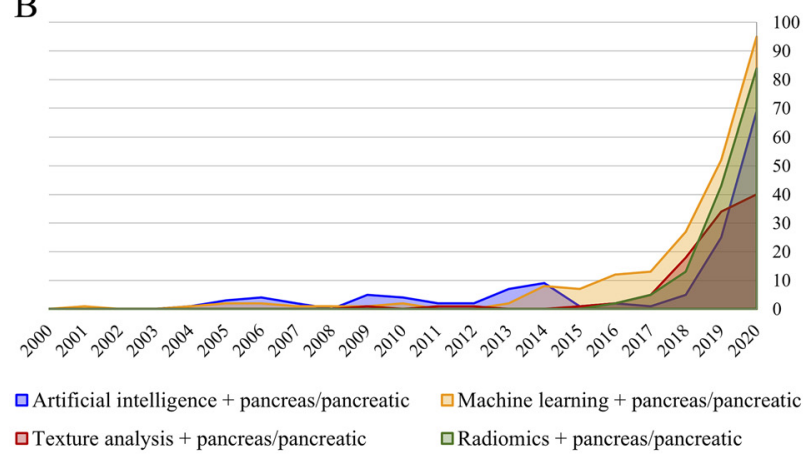

$\mathrm{C}$

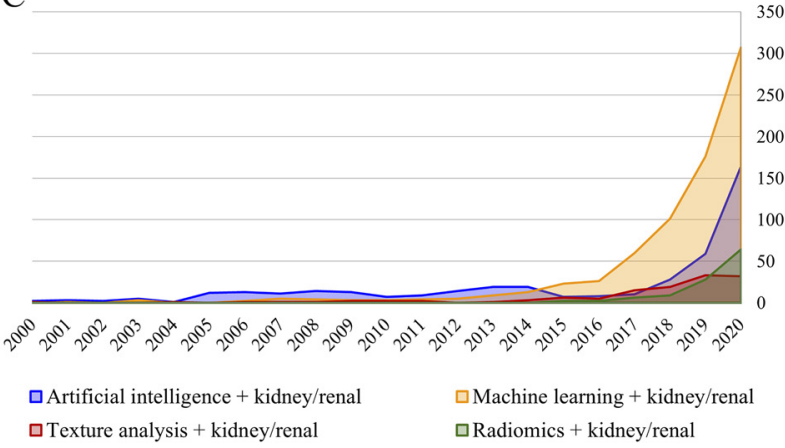

Fig. 3. The rapid increase in the number of PubMed ${ }^{\mathbb{R}}$ articles with keywords Artificial intelligence; Machine learning; Texture analysis; Radiomics published between 2000-2020.

A) The increasing number of search results of each keyword since 2000.

B) The increasing number of search results of each keyword and pancreas/pancreatic with a sharp increase from 2017.

C) The increasing number of search results of each keyword and kidney/renal with a sharp increase in research papers from 2016

3) tumors showed a significantly lower mean attenuation, higher skewness, a larger volume, a larger effective diameter, lower sphericity, lower GLCM moments and higher GLCM IDM compared to grade 1 tumors $(P<0.05)$. Furthermore, high-grade tumors showed a significantly higher GLCM entropy in arterial 2D analysis and a larger surface area in arterial 3D analysis than grade 1 tumors $(P<0.05)$. The analysis of portal venous phase scans with both $2 \mathrm{D}$ and $3 \mathrm{D}$ methods indicated that high-grade tumors are more likely to have higher skewness, lower kurtosis, higher homogeneity, larger volume, larger effective diameter, lower sphericity and lower GLCM moments compared to grade 1 tumors
$(P<0.05)$. In addition, the 3D texture analysis using portal venous phase scans revealed that high-grade tumors had a significantly larger surface area compared with grade 1 tumors $(P<0.05)$. According to a ROC analysis, the diagnostic performance of the three-dimensional analysis of the arterial phase was superior $(\mathrm{AUC}=0.774)$ to conventional $\mathrm{CT}$ features $(A U C=0.683)$ for the classification of high-grade (grades 2 and 3) vs. low-grade tumors.

Similarly, D'Onofrio et al. [16] also proved that threedimensional texture analysis of pNETs in pre-treatment CT scans holds a great promise for the prediction of tumor grade. In this study, kurtosis was the most significant radiomic parameter that yielded an AUC of 0.924 and could diagnose G3 tumors, with a cut-off value at 0.8 , with a sensitivity and a specificity of $82 \%$ and $85 \%$, respectively.

Previous reports showed promising results in the noninvasive grading of PDAC. PDACs are aggressive tumors, and surgical resection is the only curative treatment, but their prognosis is still very poor [17]. A positive resection margin, low differentiation, large tumor size, perineural and lymphovascular invasion are negative prognostic factors of overall survival (OS) in PDAC [18, 19]. Non-invasive assessment of histology characteristics in PDAC may help to identify patients who have the highest survival benefit from surgical resection. Wang et al. [20] collected preoperative pancreatic and parenchymal phase CT scans from 54 patients with either papillary or tubular PDAC and found that the histology grade was negatively correlated with the enhancement of the tumor in Spearman's rank correlation ( $\mathrm{rs}=-0.784, P<0.05)$. Although the authors attempted CTbased non-invasive assessment of the histology grade, they did not use texture analysis. Qiu et al. [13] were among the first who combined CTTA with machine learning to predict the grade in PDAC. Histogram-based first-order statistics, GLCM and GLRLM features were extracted on portal venous phase images. Among all radiomic features, only 18 parameters showed significant differences between low- and high-grade groups. A support vector machine (SVM) prediction model was constructed using these 18 texture features, which had an accuracy of $86 \%$, a sensitivity of $78 \%$ and a specificity of $95 \%$ for the discrimination of low- vs. high-grade PDAC.

\section{Prediction of the prognosis and survival of pancreatic cancer}

One of the most promising applications of radiomics is the prediction of disease outcomes. Yun et al. [21] performed a CTTA of pancreatic phase CT images to determine the predictive value of first-order texture features for pancreatic head tumors. In a multivariable Cox proportional hazards model, average (without filtration and with fine and coarse filter values of $1 \mathrm{~mm}$ and $2.5 \mathrm{~mm}$ ), SD (without filtration and with medium filter value of $2 \mathrm{~mm}$ ), contrast (with medium filter value of $1.5 \mathrm{~mm}$ ) and correlation (with fine filter value of $1 \mathrm{~mm}$ ) were found to be independent prognostic markers of shorter OS following resection. In a similar study by Khalvati et al. [22]; two GLCM parameters - sum entropy without 
filters and cluster tendency with square root filter - showed robust inter-reader reproducibility and could predict OS in patients with resected PDAC. In contrast with the above result, a study by Eilaghi et al. [23] did not find a significant association between OS of patients with PDAC and entropy, uniformity or correlation in the portal venous phase. Meanwhile, based on a Cox regression analysis, dissimilarity (coefficient $=-0.1292, P$-value $=0.045)$ and inverse difference normalized (coefficient $=71.81, P$-value $=0.046)$ were significantly linked to OS in the same patient cohort.

Cassinotto et al. [24] studied the role of CTTA in the prediction of disease-free survival (DFS) in PDAC. The authors extracted three tumor attenuation parameters and four texture heterogeneity parameters from portal venous phase CT scans. Two of the tumor heterogeneity parameters, skewness and kurtosis, showed association with early recurrence in a univariable Cox regression analysis. However, in a multivariable analysis, kurtosis showed association with early recurrence as an independent variable, while none of the tumor heterogeneity parameters were independently associated with DFS. Sandrasegaran et al. [25] collected portal venous phase images of non-resectable pancreatic cancers. The authors manually drew a polygonal ROI around the broadest cross-section of the tumor, for which they calculated the mean of positive pixels, kurtosis, skewness and entropy. According to a univariable Cox proportional hazard regression analysis, these parameters were not significantly associated with OS; however, patients with worse outcome had lower entropy, a higher mean of positive pixels (MPP) and higher kurtosis values. The MPP showed a significant association with OS in a multivariable Cox proportional hazard regression analysis, but none of the texture parameters were associated with progressionfree survival (PFS).

\section{Prediction of the molecular and genetic subtypes with radiogenomics}

Studies investigating the radiogenomics of pancreatic cancer also achieved promising results. Attiyeh et al. [26] demonstrated that radiomic analysis of preoperative portal venous phase $C T$ scans had the potential to predict the SMAD4 status and the number of altered driver mutations in PDAC. They also identified the texture features significantly associated with stromal content. In a recent study, Iwatate et al. [27] were able to predict the p53 mutation status of PDACs with good accuracy $(\mathrm{AUC}=0.705)$ by performing radiomic analysis on early and late phase CT scans. Their model showed better accuracy when a $4 \mathrm{~mm}$ wide peritumoral area was also included in the analyzed volume of interest $(\mathrm{AUC}=0.795)$. However, the model was not able to predict the PD-L1 status accurately.

\section{Radiomic analysis of renal tumors}

Renal masses are common incidental findings in abdominal CT, and a lot of them turn out to be malignant, which 
Table 2. Overview of the literature on the prediction of the histology grade of pancreatic lesions with CT texture analysis

\begin{tabular}{|c|c|c|c|c|c|}
\hline Study & Description & Imaging modality & Radiomic parameters & Classification method & Results \\
\hline Choi et al. [15] & $\begin{array}{l}\text { Prediction of the histology } \\
\text { grade of pNETs. }\end{array}$ & $\begin{array}{l}\text { Arterial phase and portal } \\
\text { venous phase CT scans }\end{array}$ & $\begin{array}{l}\text { Conventional CT findings, } \\
\text { First-order statistics, } \\
\text { Second-order GLCM texture } \\
\text { features }\end{array}$ & $\begin{array}{l}\text { Multivariate logistic } \\
\text { regression analysis } \\
\text { ROC-AUC analysis }\end{array}$ & $\begin{array}{c}\text { CT finding analysis (1 feature): } \\
\text { AUC }=0.683 \\
\text { Arterial } 2 D \text { analysis (1 feature): } \\
\text { AUC }=0.743 \\
\text { Arterial } 3 D \text { analysis ( } 2 \text { features): } \\
\text { AUC }=0.774 \\
\text { Portal venous phase } 2 D \text { analysis (2 } \\
\text { features): } \\
\text { AUC }=0.767 \\
\text { Portal venous phase } 3 D \text { analysis (2 } \\
\text { features): } \\
\text { AUC }=0.745\end{array}$ \\
\hline D'Onofrio et al. [16] & $\begin{array}{l}\text { Prediction of the histology } \\
\text { grade of pNETs. }\end{array}$ & Pancreatic phase CT scans & $\begin{array}{c}\text { qualitative imaging } \\
\text { parameters, quantitative } \\
\text { enhancement parameters } \\
\text { (ratios), first-order statistics }\end{array}$ & ROC-AUC analysis & $\begin{array}{c}\text { Kurtosis (for the diagnosis of grade } \\
\text { 3): } \\
\text { AUC }=0.924 \\
\text { Entropy (for the diagnosis of grade } \\
\text { 3): } \\
\text { AUC }=0.723\end{array}$ \\
\hline Qiu et al. [13] & $\begin{array}{c}\text { Prediction of the grade of } \\
\text { PDAC }\end{array}$ & Portal venous phase CT scans & $\begin{array}{l}\text { Histogram-based first-order } \\
\text { statistics, GLCM, GLRLM } \\
\text { texture features }\end{array}$ & $\begin{array}{c}\text { Support vector machine } \\
\text { classifier } \\
\text { Multivariate logistic } \\
\text { regression analysis }\end{array}$ & $\begin{array}{c}\text { SVM with } 18 \text { features (Model D): } \\
\text { Accuracy } 86 \%\end{array}$ \\
\hline
\end{tabular}

ROC: receiver operating characteristic curve; AUC: area under the receiver operating characteristic curve; PDAC: pancreatic ductal adenocarcinoma; pNET: pancreatic neuroendocrine tumor GLCM: gray-level co-occurrence matrix; GLRLM: gray-level run-length matrix; SVM: support vector machine 
makes accurate imaging diagnosis crucial for determining both their treatment and prognosis. Although biopsy can provide a definitive diagnosis and differentiate malignant from benign masses, its indications are restricted to cases where imaging studies alone are insufficient for therapeutic decision-making [28]. Renal cell carcinoma (RCC) is the most frequent primary neoplasia in the kidney, which typically follows an insidious course: it is asymptomatic in the early stages and only become symptomatic when it has grown to a large size. The three most common subtypes of RCC are clear cell (ccRCC), papillary (pRCC), and chromophobe (chRCC) carcinomas. Radiomics is an emerging and potentially efficient tool not only for the classification of various types of renal lesions but also to determine the histology characteristics and even the prognosis of renal tumors. Table 3 summarizes the most important reports that studied the ability of CTTA to differentiate between kidney lesions. The most recent reports that showed promising results in the prediction of histology grade of ccRCC using CTTA are summarized in Table 4.

\section{Differentiating between renal masses with radiomics}

Initial research using radiomic features has focused on differentiating solid renal tumors from benign-appearing cysts. Although prior studies have concluded that attenuation of $20 \mathrm{HU}$ or lower on the unenhanced CT images is generally diagnostic of a benign cyst, there is also significant overlap between the unenhanced densities of simple renal cysts and low-attenuation RCCs. When CTTA of unenhanced CT images was used in a study to distinguish benign cysts from low attenuation RCCs, the authors found that entropy was the most beneficial texture feature. By applying a 3.9 threshold determined by a ROC analysis, the two subtypes could be differentiated with a sensitivity of $81 \%$ and a specificity of $89 \%$ [29].

It is still highly challenging to interpret subtypes of RCC with high confidence based on visual inspection only because overlapping morphological characteristics may be present. Bata et al. [30] measured the enhancement of RCC and transitional cell carcinoma (TCC) in the corticomedullary (30-45s), nephrographic (70-90s) and excretory phases and compared it with the attenuation of the normal renal cortex. Significant differences were found in the attenuation ratios between RCC and TCC in the corticomedullary $(\mathrm{Pp}=0.040)$ and nephrographic $(P=0.004)$ phases using three ROIs of $10 \mathrm{~mm}^{2}$ size. However, when attenuation of the whole tumor was measured there was no difference in the attenuation ratios between RCC and TCC in any phases [30]. Raman et al. [31] used CTTA to differentiate ccRCC, pRCC, oncocytomas and cysts from one another. In this pilot study, CT scans were reconstructed to $3 \mathrm{~mm}$ slice thickness with $3 \mathrm{~mm}$ slice intervals. The arterial, venous and excretory phases were all used in an analysis where 10 axial slices form each lesion were matched with the corresponding slices of the other phases. The number of cases was nearly equal for each RCC type to create a balanced dataset, and an RF classifier was built, which could predict the four different histological subtypes. The trained model was able to correctly classify ccRCC with $92.5 \%$, pRCC with $100 \%$, oncocytomas with $89 \%$, and cysts with $100 \%$ accuracy. In a more recent study, Deng et al. [32] examined patients with histologically proven RCC (including ccRCC, pRCC, and chRCC) and benign tumors (oncocytoma, angiomyolipoma, and AML). A ROI was manually drawn around the largest cross-section of the tumor, and a threshold of $-50 \mathrm{HU}$ was applied to exclude gas or fat-containing pixels at the tumor margin. The entropy (odds ratio $=14.72 ; 95 \%$ confidence interval $(\mathrm{CI})=1.48-146.49 ; \quad P=0.022) \quad$ and skewness (odds ratio $=2.04 ; 95 \% \mathrm{CI}=1.07-3.89 ; P=0.031$ ) were the only parameters that could differentiate between benign and malignant tumors in a logistic regression analysis, the AUC of entropy and skewness being 0.579 $(95 \% \mathrm{CI}=0.535-0.623)$ and $0.622 \quad(95 \% \mathrm{CI}=0.578-$ $0.664)$, respectively.

Other reports tried to distinguish ccRCC from pRCC and chRCC using radiomics. Chen et al. [33] evaluated histogram-based quantitative CT features to differentiate between ccRCC and pRCC. Kidneys and tumors were manually segmented in multiple phases (non-contrast, arterial, nephrogenic and excretory), and the attenuation of the ROIs was measured in each phase. The differences in image parameters between the tumors were identified with the Pearson and Anderson-Darling tests. In comparison with pRCC, the mean and medium enhancement of ccRCC was significantly higher in the three contrast-enhanced phases. The ccRCCs also had substantially higher interquartile range and $\mathrm{SD}$ and lower skewness compared to pRCCs in the arterial and nephrographic phases. Yu et al. [34] used CTTA for the classification of common subtypes of RCC and for differentiating them from oncocytomas. In ROC analyses, kurtosis and skewness were found as excellent discriminators between clear cell RCC and oncocytoma with AUCs of 0.928 [95\% CI $=0.855-1.000]$ and 0.907 [95\% CI $=0.821-0.993]$, respectively. The median of the histogram could differentiate between papillary tumors and other subtypes with a very good AUC of 0.811 [ $95 \% \mathrm{CI}=0.735-0.887$ ]; the median was also an excellent feature to discriminate between papillary RCC and oncocytoma (AUC of 0.990 [95\% CI $=0.970-$ $1.000]$ ), and oncocytoma from all other tumors (AUC of 0.917 [95\% CI $=0.856-0.978]$ ). A linear SVM model build from CTTA features could differentiate between ccRCC, pRCC, chRCC and other tumors with an AUC of 0.91, 0.92 and 0.86 , respectively.

Lubner et al. [35] collected pre-operative unenhanced $(n=107)$, arterial phase $(n=29)$, portal venous/nephrographic phase $(n=135)$ and delayed phase $(n=99)$ CTscans of 157 patients with newly diagnosed large RCCs. A CTTA was performed on single-slice images at the level of the largest cross-section of the tumors. They found that entropy on portal venous phase was positively associated with ccRCC and was negatively associated with non-clear cell subtypes with an AUC of 0.943 [95\% CI $=0.892-0.993]$. Nuclear grade showed a significant association with the entropy and SD of the pixel distribution histogram and the 
Table 3. Overview of the most recent studies on the classification of kidney lesions using CT texture analysis

\begin{tabular}{|c|c|c|c|c|c|}
\hline Study & Description & Imaging modality & Radiomic parameters & Classification method & Results \\
\hline Kim et al. [29] & $\begin{array}{l}\text { Differentiating low- } \\
\text { attenuation RCC from renal } \\
\text { cysts }\end{array}$ & Unenhanced CT scans & $\begin{array}{l}\text { Histogram-based first-order } \\
\text { statistics }\end{array}$ & $\begin{array}{l}\text { Multivariate logistic } \\
\text { regression model } \\
\text { ROC-AUC analysis }\end{array}$ & $\begin{array}{c}\text { Entropy: } \\
\text { AUC }=0.89 \\
\text { Model combining } 3 \text { features: } \\
\text { AUC }=0.92\end{array}$ \\
\hline Raman et al. [31] & $\begin{array}{c}\text { Differentiating between } \\
\text { ccRCC, pRCC, oncocytomas } \\
\text { and cysts }\end{array}$ & $\begin{array}{c}\text { Arterial phase, portal venous } \\
\text { phase and excretory phase CT } \\
\text { scans }\end{array}$ & $\begin{array}{l}\text { Histogram-based first-order } \\
\text { statistics }\end{array}$ & Random forest classifier & $\begin{array}{c}\text { Oncocytoma vs ccRCC vs pRCC vs } \\
\text { simple cyst: } \\
\text { 3-phase: } \\
\text { 18/21 oncocytomas, } 29 / 31 \text { ccRCC, } \\
36 / 36 \text { pRCC, } 25 / 25 \text { cysts were } \\
\text { correctly classified. } \\
\text { Arterial phase: } \\
\text { 17/21 oncocytomas, } 29 / 31 \text { ccRCC, } \\
36 / 36 \text { pRCC, } 25 / 25 \text { cysts were } \\
\text { correctly classified. }\end{array}$ \\
\hline Chen et al. [33] & $\begin{array}{l}\text { Differentiating between } \\
\text { ccRCC and pRCC }\end{array}$ & $\begin{array}{l}\text { Unenhanced, arterial phase, } \\
\text { nephrographic phase, and } \\
\text { excretory phase CT scans }\end{array}$ & $\begin{array}{l}\text { Histogram-based first-order } \\
\text { statistics }\end{array}$ & ROC-AUC analysis & $\begin{array}{c}\text { mean (arterial phase) }+ \text { interquartile } \\
\text { range (nephrographic phase): } \\
\text { AUC }=0.91\end{array}$ \\
\hline Yu et al. [34] & $\begin{array}{l}\text { Differentiating between } \\
\text { ccRCC, pRCC, chRCC and } \\
\text { oncocytomas }\end{array}$ & Portal venous phase CT scans & $\begin{array}{l}\text { Histogram-based first-order } \\
\text { statistics, GLCM features, } \\
\text { GLRLM features, GLGM } \\
\text { features, Laws' features }\end{array}$ & $\begin{array}{c}\text { Support vector machine } \\
\text { classifier } \\
\text { ROC-AUC analysis }\end{array}$ & $\begin{array}{c}\text { ccRCC vs. other tumors: geometric } \\
\text { mean: AUC }=0.81 \\
\text { pRCC vs. other tumors: median: } \\
\text { AUC }=0.81 \\
\text { chRCC vs. other tumors: STD5: } \\
\text { AUC }=0.76 \\
\text { oncocytoma vs. other tumors: } \\
\text { median: AUC }=0.92\end{array}$ \\
\hline \multicolumn{6}{|c|}{$\begin{array}{l}\text { ROC: receiver operating characteristic curve; AUC: area under the receiver operating characteristic curve; RCC: renal cell carcinoma; ccRCC: clear-cell renal cell carcinoma; pRCC: papillary } \\
\text { renal cell carcinoma; chRCC; chromophobe renal cell carcinoma; GLCM: gray-level co-occurrence matrix; GLRLM: gray-level run-length matrix; GLGM: gray-level gradient matrix features }\end{array}$} \\
\hline
\end{tabular}


Table 4. Overview of the literature on the prediction of the histology grade of ccRCC with CT texture analysis

\begin{tabular}{|c|c|c|c|c|c|}
\hline Study & Description & Imaging modality & Radiomic parameters & Classification method & Results \\
\hline Feng et al. [36] & $\begin{array}{l}\text { Predicting the Fuhrman grade } \\
\text { of ccRCC }\end{array}$ & $\begin{array}{l}\text { Corticomedullary phase } \\
\text { and nephrographic phase } \\
\text { CT scans }\end{array}$ & $\begin{array}{c}\text { Histogram-based first-order } \\
\text { statistics }\end{array}$ & ROC-AUC analysis & $\begin{array}{l}\text { corticomedullary phase: } \\
\text { entropy (fine filtration): } \mathrm{AUC}=0.74 \\
\text { nephrographic phase: } \\
\text { entropy (fine filtration): AUC }=0.80 \\
\text { entropy (coarse filtration): } \\
\text { AUC }=0.83\end{array}$ \\
\hline Deng et al. [37] & $\begin{array}{l}\text { Predicting the Fuhrman grade } \\
\text { of ccRCC and differentiating } \\
\text { ccRCC from pRCC }\end{array}$ & $\begin{array}{l}\text { Portal venous phase CT } \\
\text { scans }\end{array}$ & $\begin{array}{l}\text { Histogram-based first-order } \\
\text { statistics }\end{array}$ & $\begin{array}{l}\text { Multinomial logistic } \\
\text { regression analysis } \\
\text { ROC-AUC analysis }\end{array}$ & $\begin{array}{c}\text { entropy (fine filter): } \mathrm{AUC}=0.804 \\
\text { entropy (medium filter): } \\
\text { AUC }=0.841 \\
\text { entropy (coarse filter): } \mathrm{AUC}=0.822\end{array}$ \\
\hline Cui et al. [38] & $\begin{array}{l}\text { Predicting the ISUP grade of } \\
\text { ccRCC }\end{array}$ & $\begin{array}{c}\text { Unenhanced, } \\
\text { corticomedullary phase } \\
\text { and nephrographic phase } \\
\text { CT scans }\end{array}$ & $\begin{array}{c}\text { Histogram-based first-order } \\
\text { statistics, shape features, GLCM } \\
\text { features, GLDM features, GLRLM } \\
\text { features, GTDM features, GLSZM } \\
\text { features } \\
250 \text { features selected }\end{array}$ & $\begin{array}{c}\text { Gradient boosting decision } \\
\text { tree }\end{array}$ & $\begin{array}{c}\text { External validation: } \\
\text { All features: Accuracy }=69 \% \\
\text { Nephrographic CT: Accuracy }=60 \% \\
\text { Corticomedullary CT: } \\
\text { Accuracy }=61 \% \\
\text { Unenhanced CT: } \text { Accuracy }=61 \% \text {; }\end{array}$ \\
\hline Bektas et al. [39] & $\begin{array}{l}\text { Predicting the Fuhrman grade } \\
\text { of ccRCC }\end{array}$ & $\begin{array}{l}\text { Portal venous phase } \mathrm{CT} \\
\text { scans }\end{array}$ & $\begin{array}{c}\text { Histogram-based first-order } \\
\text { statistics, Gradient features, GLCM } \\
\text { features, } \\
\text { GLRLM features, autoregressive } \\
\text { model features, } \\
\text { and Haar wavelet features }\end{array}$ & $\begin{array}{l}\text { Support vector machine, } \\
\text { multilayer perceptron, naïve } \\
\text { Bayes, } k \text {-nearest neighbours, } \\
\text { and random forest classifier }\end{array}$ & $\begin{array}{c}S V M: \text { AUC }=0.86 ; \\
\text { Accuracy }=85.1 \% \\
M L P: \text { AUC }=0.86 ; \\
\text { Accuracy }=81.4 \% \\
N B: \text { AUC }=0.811 \\
\text { Accuracy }=79.6 \% \\
k-N N: \text { AUC }=0.764 \\
\text { Accuracy }=79.6 \% \\
\text { RFC: AUC }=0.813 \\
\text { Accuracy }=75.9 \%\end{array}$ \\
\hline \multicolumn{6}{|c|}{$\begin{array}{l}\text { ROC: receiver operating characteristic curve; AUC: area under the receiver operating characteristic curve; RCC: renal cell carcinoma; ccRCC: clear-cell renal cell carcinoma; pRCC: papillary } \\
\text { renal cell carcinoma; chRCC; chromophobe renal cell carcinoma; GLCM: gray-level co-occurrence matrix; GLRLM: gray-level run-length matrix; GLDM: gray-level dependence matrix; GTDM: } \\
\text { gray-tone difference matrix; GLSZM: gray-level size-zone matrix; ICC: intra-class correlation coefficient; SVM: support vector machine; MLP: multilayer perceptron; NB: naïve Bayes; RFC: } \\
\text { random forest classifier; k-NN: k-nearest neighbor; ML: machine learning }\end{array}$} \\
\hline
\end{tabular}


mean of positive pixels. Furthermore, these features were also associated with the time to recurrence (TTR) and OS.

\section{Prediction of histology grade with CTTA}

The potential of CTTA for non-invasive grading of renal cell carcinomas, especially ccRCC, has been researched extensively. Feng et al. [36] found that entropy (with a fine Laplacian of Gaussian (LoG) filter) in the corticomedullary phase could classify low-grade vs. high-grade ccRCC with a good accuracy $(A U C=0.74)$. In contrast, entropy in the nephrographic phase (with coarse LoG filter) had an AUC of 0.83 in a similar comparison. Deng et al. [37] performed a CTTA of portal venous phase scans and found a positive correlation between Fuhrman grades and tumor diameters $(P<0.001)$ and a significant association between Fuhrman grades and entropy calculated with a coarse filter $(P<0.010)$. They also studied the ability of CTTA in the classification of RCC by histological subtypes. Although a binary logistic regression analysis showed no significant difference in tumors size between ccRCC and pRCC, the entropy of ccRCC was significantly higher compared to pRCC with fine and medium filters.

Multiple reports have investigated the advantages of second and higher-order texture features for the grading of RCCs as well. Cui et al. [38] extracted first-order, shapebased, GLCM, gray level difference matrix (GLDM), gray tone difference matrix (GTDM), GLRLM, and gray level size-zone matrix (GLSZM) features from multiphasic CT and multiparametric MRI images. Their results showed that models built from multiphasic CT and multi-sequence MRI features were superior for differentiating between low-grade and high-grade ccRCC compared with models using singlephase or single-sequence features. In a single-center retrospective study, Bektas et al. [39] analyzed radiomic parameters extracted from contrast-enhanced preoperative CT scans of 53 patients with ccRCC. The tumors were classified into low-grade (Fuhrman 1 and 2) and high-grade (Fuhrman 3 and 4) groups based on histology reports. The authors constructed SVM, multilayer perceptron (MLP), naïve Bayes (NB), k-nearest neighbors (KNN) and RF classifiers from the dataset with 10 -fold cross-validation. The SVM was the best performing model with an AUC of 0.860; it also had an accuracy of $85.1 \%$, a sensitivity of $91.3 \%$ and a specificity of $80.6 \%$ and consisted of five GLCM, three GLRLM, one gradient, and four Haar wavelet features. The authors concluded that CTTA combined with machine learning classification could be a promising method for the prediction of Fuhrman nuclear grade in ccRCC.

\section{Radiogenomics of renal cell carcinomas}

Radiogenomics analysis of renal cell carcinomas has yielded significant results in the past decade. Alessandrino et al. provide a detailed account of the most important developments in the radiogenomics of kidney tumors in an excellent review paper [40]. To summarize it in brief, the genomics of ccRCCs could be directly correlated with imaging characteristics of the tumors. Early studies mainly focused on the association between gene mutation status and conventional imaging features such as tumor margins, enhancement pattern and intratumoral calcifications etc [41, 42]. Recently, radiogenomics studies of renal tumors have also been extended to include texture analysis. In a study by Ghosh et al., the authors performed three-dimensional texture analysis on quadriphasic CT scans of patients with ccRCC and identified texture parameters that correlated with the tumors' BAP1 gene mutation status. The authors reported fair to good prediction accuracies with AUCs of $0.52 ; 0.62 ; 0.66$ and 0.71 using random forest classifiers based on sets of parameters extracted from the four contrast phases, respectively [43]. In a similar study, Kocak et al. extracted two-dimensional texture features from unenhanced CT scans and reported that a random forest classifier using only seven texture parameters was able to predict the tumor BAP1 mutation status with an AUC of 0.897 [44]. The same research group was also able to predict the tumors' PBRM1 mutation status with excellent accuracy based on texture analysis of corticomedullary phase CT scans. In this study, a random forest classifier has shown $95.0 \%$ of prediction accuracy and an AUC of 0.987 , while the accuracy and the AUC of the neural network algorithm were only $88.2 \%$ and 0.925 , respectively [45].

\section{Conclusion}

The classic morphological signs detected with routine imaging studies are frequently not specific and reproducible enough to differentiate between the many subtypes of pancreatic and renal tumors. Meanwhile, CTTA can automatically extract thousands of quantitative image features and has shown promising results in lesion characterization in both the pancreas and kidneys. The radiomic analysis can provide more detailed information on lesions compared with standard radiology reports. It could be a cost-efficient alternative to more invasive techniques to diagnose lesions with precision. Many first-order statistical parameters and higher-order texture features have already been identified as excellent classifiers able to differentiate between tumors with unique phenotypes. In the kidney where incidental lesions are commonly detected, CTTA may have the potential to distinguish between benign and malignant lesions with high accuracy. Radiogenomics studies have also successfully correlated a subset of CTTA features with specific genetic mutations in pancreatic cancer. Besides, CTTA has also proved its value for the prediction of survival and tumor recurrence. In the pancreas, where curative resection is feasible only in a small percentage of the tumor cases, CTTA can promote more objective patient selection and therapeutic decision-making.

Conflict of interest: The authors have no conflict of interest to disclose.

Authors' contribution: SS - Conceptualization, Drafting of the manuscript, Proofreading 
VF - Conceptualization, Drafting of the manuscript, Proofreading

BKB - Drafting of the manuscript, Preparing the Figures

BF - Data Curation, Drafting of the manuscript

AT - Data Curation, Drafting of the manuscript

VO - Data Curation, Drafting of the manuscript

VB - Drafting of the manuscript, Critical revision

PNK - Conceptualization, Critical revision

All authors reviewed the final version of the manuscript and agreed to submit it to IMAGING for publication.

Funding sources: PNK (Bolyai 386/2017) was supported by the János Bolyai Research Scholarship of the Hungarian Academy of Sciences (https://mta.hu/bolyai-osztondij). $\mathrm{BKB}, \mathrm{AT}$ and $\mathrm{PNK}$ received financial support from the GINOP 2-2-18 grant. The funders had no role in study design, data collection and analysis, decision to publish, or preparation of the manuscript.

\section{ACKNOWLEDGEMENTS}

Not applicable.

\section{REFERENCES}

[1] Castellano G, Bonilha L, Li LM, Cendes F: Texture analysis of medical images. Clin Radiol 2004; 59(12): 1061-9.

[2] Aerts HJ, Velazquez ER, Leijenaar RT, Parmar C, Grossmann P, Carvalho S, et al.: Decoding tumour phenotype by noninvasive imaging using a quantitative radiomics approach. Nat Commun 2014; 5: 4006.

[3] Lambin P, Leijenaar RTH, Deist TM, Peerlings J, de Jong EEC, van Timmeren J, et al.: Radiomics: the bridge between medical imaging and personalized medicine. Nat Rev Clin Oncol 2017; 14(12): 749-62.

[4] Dalal V, Carmicheal J, Dhaliwal A, Jain M, Kaur S, Batra SK: Radiomics in stratification of pancreatic cystic lesions: machine learning in action. Cancer Lett 2020; 469: 228-37.

[5] Zwanenburg A, Leger S, Vallières M, Löck S: Image biomarker standardisation initiative. arXiv preprint arXiv:1612.07003. 2016.

[6] Gerlinger M, Rowan AJ, Horswell S, Math M, Larkin J, Endesfelder $D$, et al.: Intratumor heterogeneity and branched evolution revealed by multiregion sequencing. N Engl J Med 2012; 366(10): 883-92.

[7] Nelson DA, Tan T-T, Rabson AB, Anderson D, Degenhardt K, White E: Hypoxia and defective apoptosis drive genomic instability and tumorigenesis. Genes Dev 2004; 18(17): 2095-107.

[8] Chu LC, Park S, Kawamoto S, Fouladi DF, Shayesteh S, Zinreich ES, et al.: Utility of CT radiomics features in differentiation of pancreatic ductal adenocarcinoma from normal pancreatic tissue. AJR Am J Roentgenol 2019; 213(2): 349-57.

[9] Ren S, Zhang J, Chen J, Cui W, Zhao R, Qiu W, et al.: Evaluation of texture analysis for the differential diagnosis of mass-forming pancreatitis from pancreatic ductal adenocarcinoma on contrastenhanced CT images. Front Oncol 2019; 9: 1171.

[10] Reinert CP, Baumgartner K, Hepp T, Bitzer M, Horger M: Complementary role of computed tomography texture analysis for differentiation of pancreatic ductal adenocarcinoma from pancreatic neuroendocrine tumors in the portal-venous enhancement phase. Abdom Radiol (NY) 2020; 45(3): 750-8.

[11] Li J, Lu J, Liang P, Li A, Hu Y, Shen Y, et al.: Differentiation of atypical pancreatic neuroendocrine tumors from pancreatic ductal adenocarcinomas: using whole-tumor CT texture analysis as quantitative biomarkers. Cancer Med 2018; 7(10): 4924-31.

[12] Polk SL, Choi JW, McGettigan MJ, Rose T, Ahmed A, Kim J, et al.: Multiphase computed tomography radiomics of pancreatic intraductal papillary mucinous neoplasms to predict malignancy. World J Gastroenterol 2020; 26(24): 3458-71.

[13] Qiu W, Duan N, Chen X, Ren S, Zhang Y, Wang Z, et al.: Pancreatic ductal adenocarcinoma: machine learning-based quantitative computed tomography texture analysis for prediction of histopathological grade. Cancer Manag Res 2019; 11: 9253-64.

[14] Choe J, Kim KW, Kim HJ, Kim DW, Kim KP, Hong S-M, et al.: What is new in the 2017 World Health Organization classification and 8th American Joint Committee on cancer staging system for pancreatic neuroendocrine neoplasms? Korean J Radiol 2019; 20(1): 5-17.

[15] Choi TW, Kim JH, Yu MH, Park SJ, Han JK: Pancreatic neuroendocrine tumor: Prediction of the tumor grade using CT findings and computerized texture analysis. Acta Radiol 2018; 59(4): 383-92.

[16] D'Onofrio M, Ciaravino V, Cardobi N, De Robertis R, Cingarlini $\mathrm{S}$, Landoni L, et al.: CT enhancement and 3D texture analysis of pancreatic neuroendocrine neoplasms. Sci Rep 2019; 9(1): 2176.

[17] Chakraborty S, Singh S: Surgical resection improves survival in pancreatic cancer patients without vascular invasion- a population based study. Ann Gastroenterol 2013; 26(4): 346-52.

[18] Chen JWC, Bhandari M, Astill DS, Wilson TG, Kow L, BrookeSmith $M$, et al.: Predicting patient survival after pancreaticoduodenectomy for malignancy: Histopathological criteria based on perineural infiltration and lymphovascular invasion. HPB (Oxford) 2010; 12(2): 101-8.

[19] Han SH, Heo JS, Choi SH, Choi DW, Han IW, Han S, et al.: Actual long-term outcome of $\mathrm{T} 1$ and $\mathrm{T} 2$ pancreatic ductal adenocarcinoma after surgical resection. Int J Surg 2017; 40: 68-72.

[20] Wang SH, Sun YF, Liu Y, Zhou Y, Liu Y: CT contrast enhancement correlates with pathological grade and microvessel density of pancreatic cancer tissues. Int J Clin Exp Pathol 2015; 8(5): 5443-9.

[21] Yun G, Kim YH, Lee YJ, Kim B, Hwang JH, Choi DJ: Tumor heterogeneity of pancreas head cancer assessed by CT texture analysis: Association with survival outcomes after curative resection. Sci Rep 2018; 8(1): 7226.

[22] Khalvati F, Zhang Y, Baig S, Lobo-Mueller EM, Karanicolas P, Gallinger S, et al.: Prognostic value of CT radiomic features in resectable pancreatic ductal adenocarcinoma. Sci Rep 2019; 9(1): 5449.

[23] Eilaghi A, Baig S, Zhang Y, Zhang J, Karanicolas P, Gallinger S, et al.: CT texture features are associated with overall survival in pancreatic ductal adenocarcinoma - a quantitative analysis. BMC Med Imaging 2017; 17(1): 38.

[24] Cassinotto C, Chong J, Zogopoulos G, Reinhold C, Chiche L, Lafourcade J-P, et al.: Resectable pancreatic adenocarcinoma: Role of CT quantitative imaging biomarkers for predicting pathology and patient outcomes. Eur J Radiol 2017; 90: 152-8. 
[25] Sandrasegaran K, Lin Y, Asare-Sawiri M, Taiyini T, Tann M: CT texture analysis of pancreatic cancer. Eur Radiol 2019; 29(3): 1067-73.

[26] Attiyeh MA, Chakraborty J, McIntyre CA, Kappagantula R, Chou Y, Askan G, et al.: CT radiomics associations with genotype and stromal content in pancreatic ductal adenocarcinoma. Abdom Radiol (NY) 2019; 44(9): 3148-57.

[27] Iwatate Y, Hoshino I, Yokota H, Ishige F, Itami M, Mori Y, et al.: Radiogenomics for predicting p53 status, PD-L1 expression, and prognosis with machine learning in pancreatic cancer. Br J Cancer 2020.

[28] Caoili EM, Davenport MS: Role of percutaneous needle biopsy for renal masses. Semin Intervent Radiol 2014; 31(1): 20-6.

[29] Kim NY, Lubner MG, Nystrom JT, Swietlik JF, Abel EJ, Havighurst TC, et al.: Utility of CT texture analysis in differentiating low-attenuation renal cell carcinoma from cysts: a Biinstitutional retrospective study. AJR Am J Roentgenol 2019; 213(6): 1259-66.

[30] Bata P, Tarnoki DL, Tarnoki AD, Novak PK, Gyebnar J, Kekesi D, et al.: Transitional cell and clear cell renal carcinoma: Differentiation of distinct histological types with multiphase CT. Acta Radiol 2014; 55(9): 1112-9.

[31] Raman SP, Chen Y, Schroeder JL, Huang P, Fishman EK: CT texture analysis of renal masses: pilot study using random forest classification for prediction of pathology. Acad Radiol 2014; 21(12): 1587-96.

[32] Deng Y, Soule E, Cui E, Samuel A, Shah S, Lall C, et al.: Usefulness of CT texture analysis in differentiating benign and malignant renal tumours. Clin Radiol 2020; 75(2): 108-15.

[33] Chen F, Huhdanpaa H, Desai B, Hwang D, Cen S, Sherrod A, et al.: Whole lesion quantitative CT evaluation of renal cell carcinoma: Differentiation of clear cell from papillary renal cell carcinoma. SpringerPlus 2015; 4(1): 66.

[34] Yu H, Scalera J, Khalid M, Touret A-S, Bloch N, Li B, et al.: Texture analysis as a radiomic marker for differentiating renal tumors. Abdom Radiol (NY) 2017; 42(10): 2470-8.

[35] Lubner MG, Stabo N, Abel EJ, Del Rio AM, Pickhardt PJ: CT textural analysis of large primary renal cell carcinomas: pretreatment tumor heterogeneity correlates with histologic findings and clinical outcomes. AJR Am J Roentgenol 2016; 207(1): 96-105.
[36] Feng Z, Shen Q, Li Y, Hu Z: CT texture analysis: A potential tool for predicting the Fuhrman grade of clear-cell renal carcinoma. Cancer Imaging 2019; 19(1): 6.

[37] Deng Y, Soule E, Samuel A, Shah S, Cui E, Asare-Sawiri M, et al.: CT texture analysis in the differentiation of major renal cell carcinoma subtypes and correlation with Fuhrman grade. Eur Radiol 2019; 29(12): 6922-9.

[38] Cui E, Li Z, Ma C, Li Q, Lei Y, Lan Y, et al.: Predicting the ISUP grade of clear cell renal cell carcinoma with multiparametric MR and multiphase CT radiomics. Eur Radiol 2020; 30(5): 2912-21.

[39] Bektas CT, Kocak B, Yardimci AH, Turkcanoglu MH, Yucetas U, Koca SB, et al.: Clear cell renal cell carcinoma: machine learningbased quantitative computed tomography texture analysis for prediction of Fuhrman nuclear grade. Eur Radiol 2019; 29(3): 1153-63.

[40] Alessandrino F, Shinagare AB, Bossé D, Choueiri TK, Krajewski KM: Radiogenomics in renal cell carcinoma. Abdom Radiol (NY) 2019; 44(6): 1990-8.

[41] Karlo CA, Di Paolo PL, Chaim J, Hakimi AA, Ostrovnaya I, Russo P, et al.: Radiogenomics of clear cell renal cell carcinoma: Associations between CT imaging features and mutations. Radiology 2014; 270(2): 464-71.

[42] Shinagare AB, Vikram R, Jaffe C, Akin O, Kirby J, Huang E, et al.: Radiogenomics of clear cell renal cell carcinoma: Preliminary findings of the cancer genome atlas-renal cell carcinoma (TCGARCC) imaging research group. Abdom Imaging 2015; 40(6): 1684-92.

[43] Ghosh P, Tamboli P, Vikram R, Rao A: Imaging-genomic pipeline for identifying gene mutations using three-dimensional intra-tumor heterogeneity features. J Med Imaging (Bellingham) 2015; 2(4): 041009.

[44] Kocak B, Durmaz ES, Kaya OK, Kilickesmez O: Machine learningbased unenhanced CT texture analysis for predicting BAP1 mutation status of clear cell renal cell carcinomas. Acta Radiologica 2019; 61(6): 856-64.

[45] Kocak B, Durmaz ES, Ates E, Ulusan MB: Radiogenomics in clear cell renal cell carcinoma: machine learning-based high-dimensional quantitative CT texture analysis in predicting PBRM1 mutation status. AJR Am J Roentgenol 2019; 212(3): W55-63. 